\title{
Effect of surface modification and hybridization on dynamic mechanical properties of Roystonea regia/glass-epoxy composites
}

\author{
GOVARDHAN GOUD* and R N RAO \\ Department of Mechanical Engineering, National Institute of Technology, Warangal 506 004, India
}

MS received 4 September 2011; revised 28 February 2012

\begin{abstract}
The paper evaluates effect of fibre surface modification and hybridization on dynamic mechanical properties of Roystonea regia/epoxy composites. Surface modification involved alkali and silane treatments. Alkali treatment proved to be more effective on dynamic mechanical properties as compared to silane treatment. Storage and loss modulus values increased after treatments with simultaneous decrease in $\tan \delta$ values. Roystonea regia and glass fibres were used together with varying proportions as reinforcement in epoxy matrix to study the hybridization effect on dynamic mechanical properties. Storage and loss modulus values increased with increase in glass fibre content whereas $\tan \delta$ values were found to decrease. Scanning electron microscopy of tensile fractured surfaces was carried out to study the interface adhesion of different composites.
\end{abstract}

Keywords. Epoxy; Roystonea regia; glass; surface modification; hybridization; dynamic mechanical properties.

\section{Introduction}

Polymer composites reinforced with high-performance fibres such as carbon, boron, Kevlar etc were developed and successfully used in industries. But after service, the treatment and disposal of these materials as solid waste has become a serious problem. To overcome this, efforts are being made to use environment friendly materials. It is in this context that natural fibre composites assumed significance (Bledzki and Gassan 1999). Natural fibre composites, apart from being biodegradable and renewable also offer several other advantages such as light weight, low cost, high specific strength, high modulus, reduced tool wear and safe manufacturing process when compared to synthetic fibre composites. Several researchers carried out investigations and developed natural fibre-reinforced composites. Pothan et al (1997) investigated on mechanical, failure and aging characteristics of short banana fibre reinforced polyester composites and Manikandan et al (1996) studied tensile properties of short sisal fibre reinforced polystyrene composites. Pickering et al (2007) carried out the optimization of industrial hemp fibre for composites.

Mechanical properties of natural fibre composites mainly depend on the fibre/matrix interface and its ability to transfer the stress from the matrix to fibre. But it is observed that poor interface adhesion exists between natural fibre and polymer matrix due to which natural fibre composites exhibit inferior strength. The main reason for poor compatibility is polymer matrix which is hydrophobic and nonpolar and the natural fibres are hydrophilic and have polar

\footnotetext{
*Author for correspondence (pgovardhan0@yahoo.com)
}

groups in their structure. Based on their findings, investigators suggest surface modification methods like alkali and silane coupling treatment of the fibre to improve interfacial bonding. Sinha and Rout (2009) studied effect of alkali treatment on jute fibre composites and reported improvement in flexural properties. Rout et al (2001) reported that after $2 \%$ alkali treatment coir fibre polyester composites showed improved tensile properties. There is a $15 \%$ increase in tensile strength after alkali treatment of unidirectional sisal reinforced epoxy composites. Huda et al (2008) in their investigation pointed out that surface treated fibre reinforced composites showed superior mechanical properties than untreated fibre reinforced composites. Bisanda and Ansell (1991) studied effect of silanes on mechanical properties of sisal-epoxy composites and found considerable enhancement in compressive strength. Natural fibres are widely treated with silane coupling agent because it will improve the degree of cross linking in the interface region and offers a suitable bonding along with the creation of higher surface area. This will lead to improvement in overall performance of the composites (Rong et al 2001). Alkali treatment, by removing the hemicelluloses, lignin and waxes from the fibre would convert the fibre surface coarser leading to better interface between matrix and fibre. Alkalization also causes fibrillation i.e. breaking of fibre bundles into smaller fibres which would increase the effective surface area available for wetting by the matrix material ( $\mathrm{Li}$ et al 2000). After fibrillation due to the reduced diameter of the fibre, aspect ratio of the fibre increases and yields rough surface topography which in turn offers better fibre/matrix interface. This results in obtaining the enhanced properties. Also to overcome the limitations of lower modulus, lower strength and poor moisture resistance and to obtain 
great diversity of material properties, hybrid composites have been conceived wherein two or more fibres are reinforced in a single matrix. In hybrid composites higher performance of synthetic fibre and environmental advantages of natural fibres would be combined. These days along with natural fibres, glass fibres are widely used in hybrid composites due to their higher strength, light weight, dimensional stability, resistance to corrosion and electricity. Several investigators developed hybrid composites by reinforcing natural fibres with glass and reported improved properties. Hybrid effect of glass and OPEFB fibre was investigated by Srikala et al (2002) and the study revealed overall improvement in the performance after hybridization. Raghavendra Rao et al (2010) found superior flexural and compressive properties by hybridizing the bamboo and glass fibres with epoxy.

Dynamical mechanical analysis (DMA) is a very useful technique to study the mechanical behaviour of material on the basis of viscoelasticity. The test reveals response of the material when it is deformed under the influence of sinusoidal or periodic stress. The important parameters in DMA tests are: (i) storage modulus which indicates stiffness behaviour and load bearing capacity of the material, (ii) loss modulus which indicates the energy dissipated in the form of heat by the material and (iii) mechanical damping or tan $\delta$ which is the ratio of loss modulus to storage modulus and very much depends on degree of molecular mobility in the material.

\section{Experimental}

\subsection{Materials}

The matrix system used is an epoxy resin (Lapox-12) and hardener (k-6) both supplied by Atul Limited, Gujarat, India. To study the effect of surface modification, unidirectional Roystonea regia (royal palm) fibre was used as reinforcement which was collected from the foliage of locally available royal palm tree through the water retting and mechanical extraction. In hybrid composites both $E$-glass and Roystonea regia fibres were used in short form $(5-8 \mathrm{~mm})$ as reinforcement in epoxy matrix.

\subsection{Fibre extraction}

From the foliage sheath, the leaves and leaf stem were removed and the sheath was dried for three days in shade. In the next step, it was immersed in water retting tank for three weeks followed by hand rubbing and rinsing in water till the unwanted greasy material was dissolved and fine fibre was extracted. Finally the extracted fibre was once again washed thoroughly in plenty of clean water to remove the surplus waste. Continuous fibre was obtained with a length up to 1.5 meters. The obtained fibre was dried under sun for one week. The average diameter of the fibre used for the composite preparation was between 0.2 and $0.3 \mathrm{~mm}$.

\subsection{Alkali treatment}

The dry fibre was treated with $5 \%$ solution of $\mathrm{NaOH}$ for $2 \mathrm{~h}$ to remove the unwanted soluble cellulose, hemi cellulose, pectin, lignin etc from the fibre. The fibre to solution weight ratio was maintained at 1:25. After $2 \mathrm{~h}$, the fibre was washed thoroughly in distilled water to remove excess of $\mathrm{NaOH}$ and dried at $60{ }^{\circ} \mathrm{C}$ for $24 \mathrm{~h}$.

\subsection{Silane treatment}

The coupling agent 3-aminopropyl tri-ethoxy silane was diluted to $1 \%(\mathrm{v} / \mathrm{v})$ in acetone and the dry fibre was dipped in diluted coupling agent solution for $3 \mathrm{~h}$. Fibre was then washed and kept in air for 3 days. Finally, the fibre was dried in an oven at $80^{\circ} \mathrm{C}$ for $12 \mathrm{~h}$.

\subsection{Composite preparation}

The mould box was prepared with dimensions of 200 (L) $\times 150(\mathrm{~W}) \times 3.0(\mathrm{~T}) \mathrm{mm}$. Matrix was prepared by mixing the hardener to epoxy. The epoxy and hardener ratio was maintained at 10:1. Mould box was loaded with appropriate quantities of matrix and fibres and finally the compression pressure of $170 \mathrm{KN}$ was applied evenly to achieve a uniform thickness of $3 \mathrm{~mm}$ and cured for $24 \mathrm{~h}$ at room temperature. To study the effect of surface treatments, 3 types of composites were prepared. They are untreated (UT), alkali treated (AT) and silane coupling agent treated (CT) unidirectional Roystonea regia fibre reinforced composites. In all the composites, percentage of fibre was $20 \%$ by weight. Also to investigate hybridization effect, five types of composites were prepared by varying Roystonea regia and glass fibre content. The sample designation and the content details are listed in

Table 1. Loss modulus and damping values of composites.

\begin{tabular}{lcccc}
\hline Sample & $\begin{array}{c}T_{\mathrm{g}}\left({ }^{\circ} \mathrm{C}\right) \text { obtained from } \\
\text { loss modulus curve }\end{array}$ & Loss modulus $(\mathrm{MPa})$ & $\begin{array}{c}\text { Damping factor } \\
\left(\tan \delta_{\max }\right)\end{array}$ & $\begin{array}{c}\text { Temperature }\left({ }^{\circ} \mathrm{C}\right) \text { at } \\
\tan \delta_{\max }\end{array}$ \\
\hline NE & 65.61 & 327.36 & 0.84 & 83.4 \\
UT & 69.36 & 361.03 & 0.66 & 76.9 \\
CT & 76.86 & 380.97 & 0.63 & 88.6 \\
AT & 84.38 & 416.30 & 0.60 & 73.1 \\
\hline
\end{tabular}


Table 2. Designation and composition of composites.

\begin{tabular}{lccc}
\hline Designation & Epoxy (\%wt.) & Roystonea regia $(\% \mathrm{wt})$. & Glass $(\% \mathrm{wt})$. \\
\hline $\mathrm{NE}$ & 100 & 0 & 0 \\
0G & 80 & 20 & 0 \\
$25 \mathrm{G}$ & 80 & 15 & 05 \\
$50 \mathrm{G}$ & 80 & 10 & 10 \\
$75 \mathrm{G}$ & 80 & 05 & 15 \\
$100 \mathrm{G}$ & 80 & 0 & 20 \\
\hline
\end{tabular}

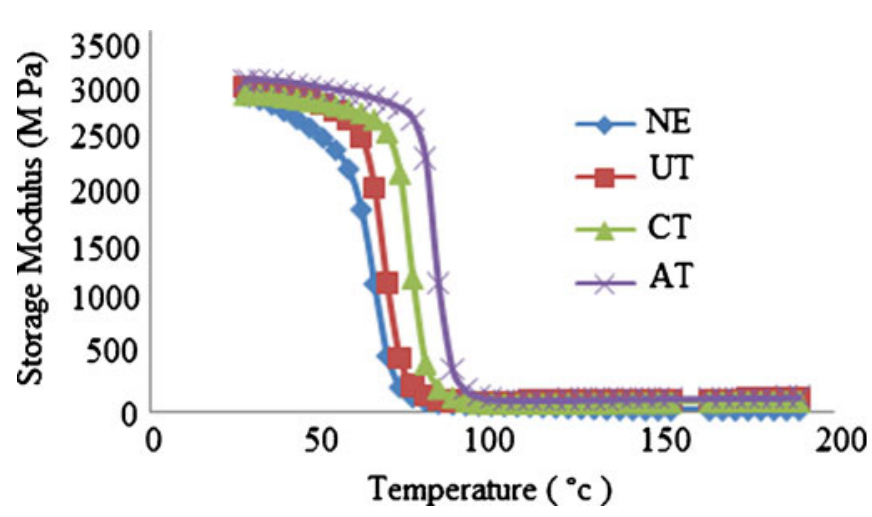

Figure 1. Effect of surface treatment on storage modulus at different temperatures.

table 2. However, neat epoxy (NE) plate was also fabricated for comparison.

\subsection{DMA testing}

DMA testing was carried out with rectangular specimens of size $60 \times 10 \times 3 \mathrm{~mm}$ on a Universal V4.5A TA-instrument at a temperature range of $25-180^{\circ} \mathrm{C}$. Testing was carried out at a heating rate of $3^{\circ} \mathrm{C}$ and frequency of $1 \mathrm{~Hz}$ in three-point bending mode.

\section{Results and discussion}

\subsection{Effect of surface modification on dynamic mechanical properties}

3.1a Storage modulus: Storage modulus is maximum energy stored in the material in one cycle of operation (Jacob et al 2006). It indicates stiffness behaviour and load bearing capacity of composite material. Figure 1 shows temperature dependence of dynamic storage modulus of the neat epoxy (NE), untreated (UT), coupling agent treated (CT) and alkali treated (AT) fibre composites. Figure 1 shows that storage modulus of fibre reinforced composites is higher than unfilled neat epoxy. This is due to the fact that neat epoxy, as it comprises of only the matrix, gives the material more flexibility resulting in a decreased stiffness and hence low storage modulus. When Roystonea regia fibres are incorporated in the epoxy matrix, stiffness of the composites increases resulting in an enhanced storage modulus. At the same time, addition of fibres to matrix allows greater stress transfer at interface which consequently increases the storage modulus.

Among the Roystonea regia fibre reinforced composites, treated fibre reinforced composites have shown higher storage modulus than the untreated fibre composites. In untreated fibre composites, higher hydrophilic nature of the lingocellulosic royal palm fibres induce poor wettability and higher presence of moisture at fibre-matrix interface promotes the formation of voids leading to lower stiffness and strength which results in lower storage modulus. Both silane and alkali surface treatments proved to be effective in increasing the storage modulus. Coupling agent improves the degree of cross linking in the interface region and offers a suitable bonding along with the creation of higher surface area. This results into better interface between matrix and fibre which in turn leads to increased storage modulus. Alkali treatment had shown more pronounced effect on storage modulus when compared with silane coupling treatment. In alkali treatment hemi cellulose and lignin contents will be removed from the fibre and produces rough surface (coarse) on the fibre leading to better interface between matrix and fibre. Alkalization also causes fibrillation i.e. breaking of fibre bundles into smaller fibres which would increase the effective surface area available for wetting by the matrix material. After fibrillation due to the reduced diameter of the fibre, the aspect ratio of the fibre increases and yields rough surface topography which in turn offers better fibre/matrix interface. This results in obtaining enhanced stiffness and higher storage modulus. The effect of alkali treatment in modifying surface of fibre can be observed by scanning electron microscopy (SEM) as shown in figures 2(a) and 2(b). The alkali treated fibre has become coarser (see figure 2(b)) when compared with the untreated fibre (see figure 2(a)). For all the composites, it is observed that variation of storage modulus is more prominent in glassy region when compared with the rubbery region.

3.1b Loss modulus: Loss modulus is proportional to the amount of energy that has been dissipated as heat by the 


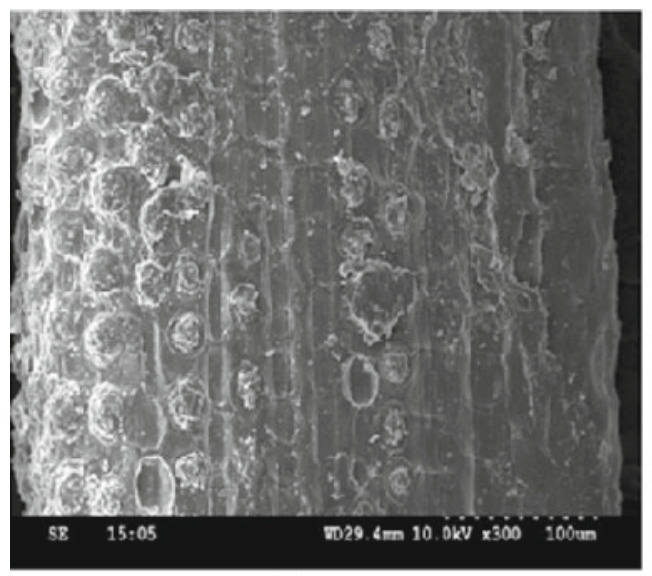

(a)

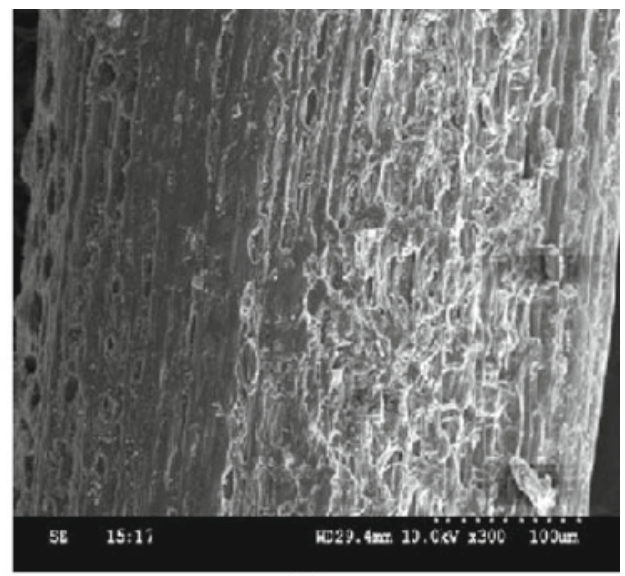

(b)

Figure 2. SEM micrographs of (a) untreated fibre and (b) alkali treated fibre.

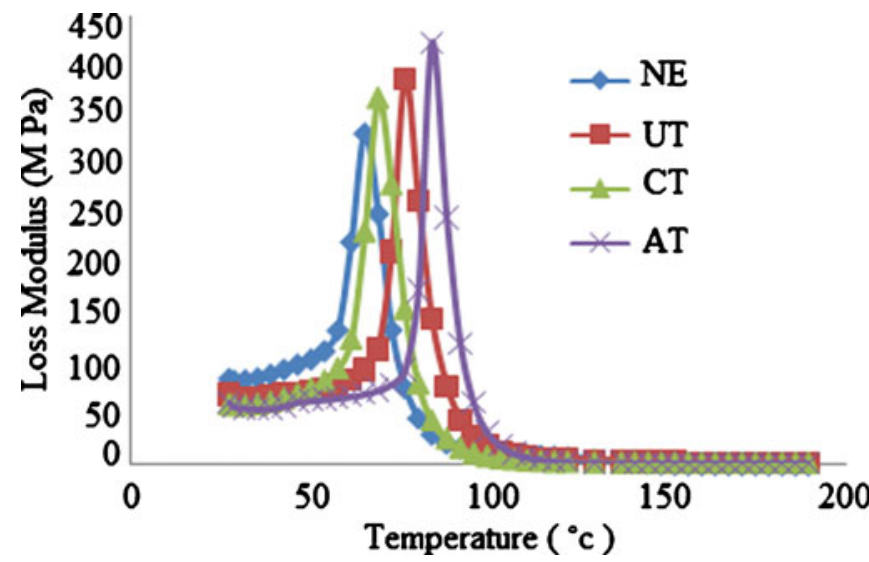

Figure 3. Effect of surface treatment on loss modulus at different temperatures.

sample. The maximum heat dissipation occurs at the temperature where loss modulus is maximum which indicates glass transition $\left(T_{\mathrm{g}}\right)$ of the material. Higher $T_{\mathrm{g}}$ values would mean less free mobility of free amorphous phase in the composites. Loss modulus for neat epoxy, untreated and treated fibre composites have been plotted as a function of temperature in figure 3 and table 1 . The $T_{\mathrm{g}}$ of all fibre reinforced composites shifted to higher temperatures when compared with neat epoxy. It is due to the decreased mobility of matrix chains owing to the presence of the fibres. Compared to untreated fibre composite, treated fibre composites have shown more $T_{\mathrm{g}}$ and loss modulus values. The increase of $T_{\mathrm{g}}$ in silane coupling and alkali treated fibre composites is associated with the decreased mobility of the matrix chains, which indicates enhanced interfacial adhesion between the fibre and the matrix after treatments. Alkali treatment has shown more pronounced effect on $T_{\mathrm{g}}$ and loss modulus values when compared with silane treatment.

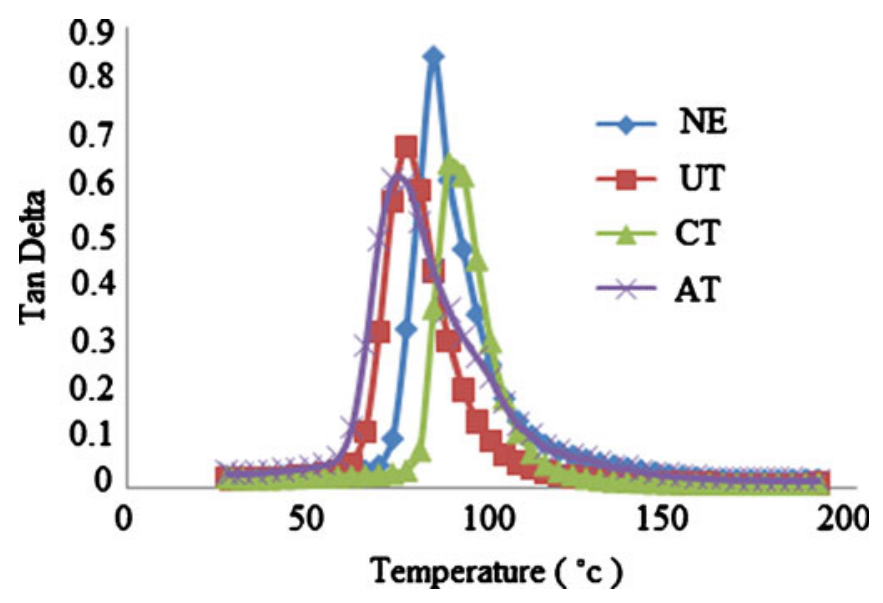

Figure 4. Effect of surface treatment on $\tan \delta$ at different temperatures.

3.1c $\operatorname{Tan} \delta$ : Mechanical damping $(\tan \delta$ ) is the ratio of loss modulus to storage modulus and is related to the molecular mobility in polymer material. Tan $\delta$ relates to the impact resistance of the material and associated with the movement of small groups and chains of molecules within the polymer structure. Hence higher the $\tan \delta$ peak value greater would be the molecular mobility (Kuzak and Shanmugam 1999). The variation of $\tan \delta$ of the neat epoxy, different composites as a function of temperature is shown in figure 4. From figure 4 and table 1 , it is evident that neat epoxy has the highest $\tan \delta$ peak value indicating higher degree of mobility and therefore, better damping properties. Fibre reinforced composites have shown decreased damping values due to the fact that incorporation of fibres would act as barriers and check mobility of the matrix chains. Another reason for the lower damping values of fibre reinforced composites is reduction in matrix volume required for the dissipation of vibration energy. Also values indicate that composites reinforced with chemically treated fibres (CT and 


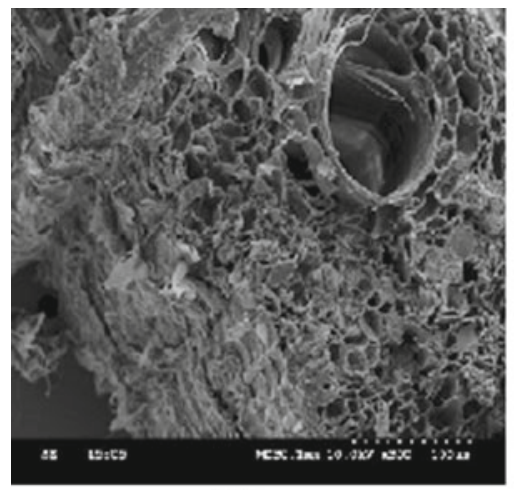

(a)

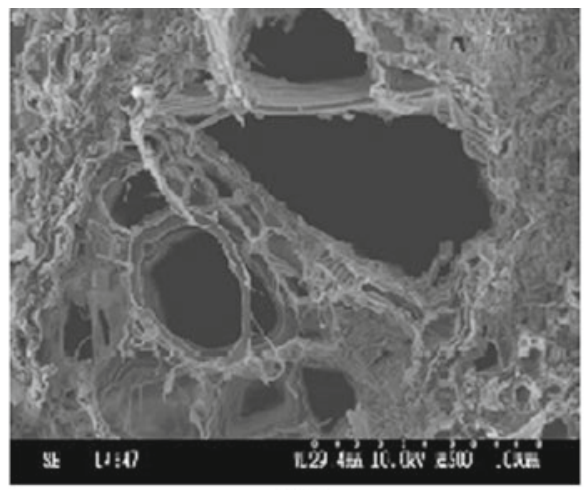

(b)

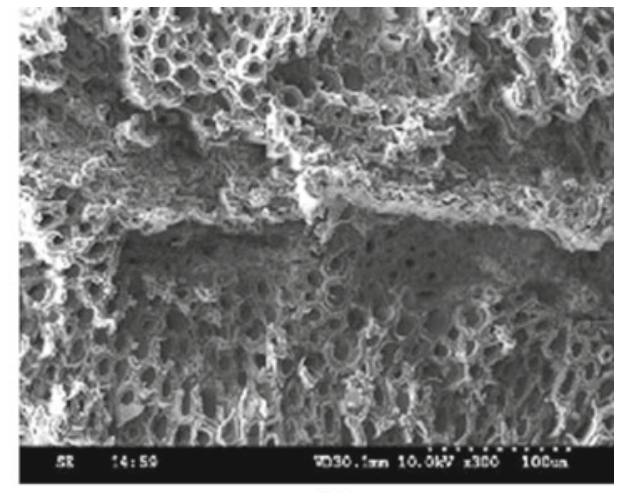

(c)

Figure 5. SEM pictures of tensile fractured surfaces (a) untreated fibre composite, (b) silane treated fibre composite and (c) alkali treated fibre composite [all at $20 \%$ fibre content (\%wt)].

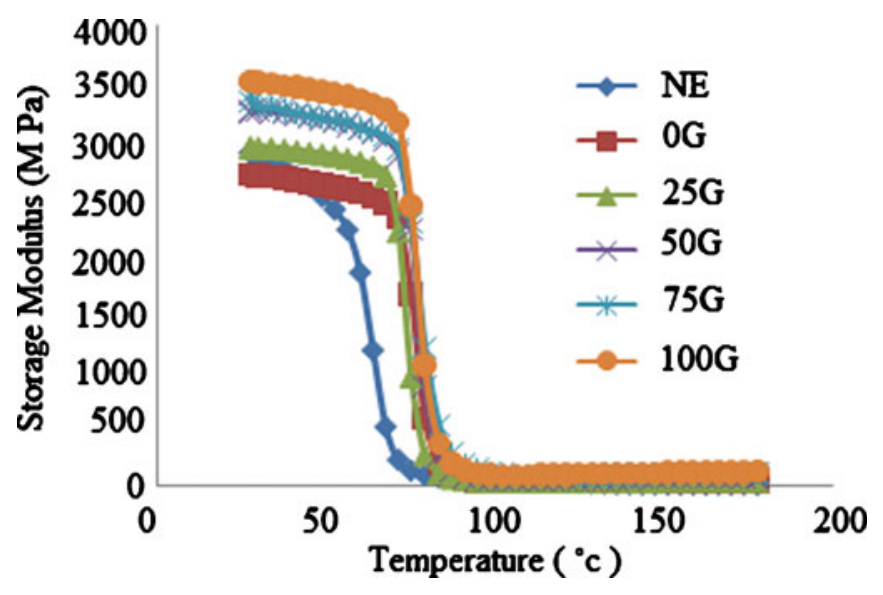

Figure 6. Effect of hybridization on storage modulus at different temperatures.

AT) have shown decreased $\tan \delta$ values than untreated composites because after silane and alkali treatments strong and rigid interface develops between fibre and matrix. This would reduce the molecular mobility in the interface region. Also surface treatments improve the degree of cross linking in interface area which hinders the molecular mobility resulting in low damping values. Among the treated fibre composites, silane treated fibre composites have shown higher damping value than the alkali treated fibre composites indicating lower fibre/matrix interface when compared with the alkali treated fibre composites. The fracture morphology of tensile specimens was shown to compare the fibre/matrix interface of untreated, silane treated and alkali treated fibre composites as shown in figure $5(\mathrm{a}-\mathrm{c})$. SEM pictures indicate stronger interface of treated fibre composites (figures 5(b) and (c)) than untreated fibre composite (figure 5(a)). Also from the figures it can be observed that alkali treated fibre composites have stronger fibre/matrix interface (figure 5(c)) than the silane treated fibre composites (figure 5(b)).

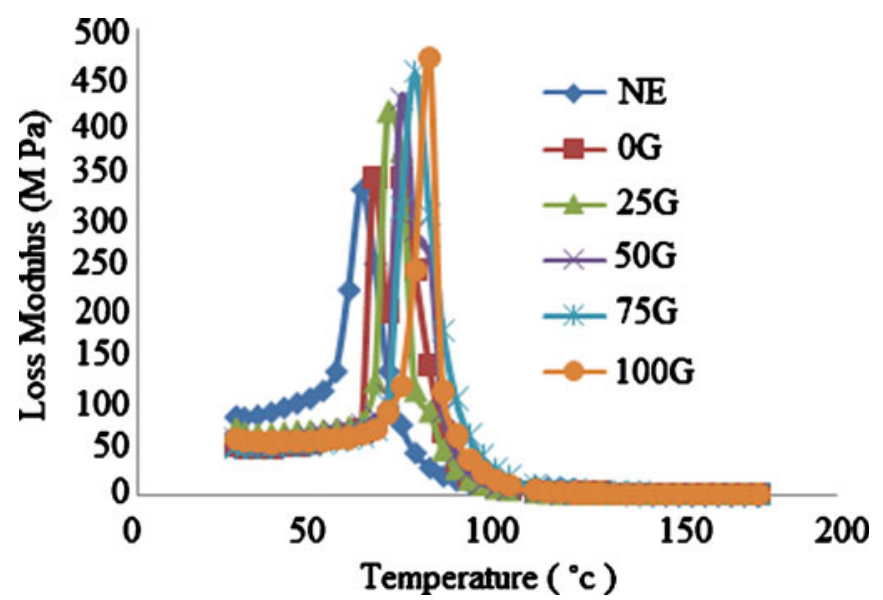

Figure 7. Effect of hybridization on loss modulus at different temperatures.

\subsection{Effect of hybridization on dynamic mechanical properties}

3.2a Storage modulus: Figure 6 shows temperature dependence of storage modulus of the neat epoxy (NE) and different hybrid composites with varying Roystonea regia and glass fibre contents. From the figure it is evident that storage modulus followed the order NE $<0 \mathrm{G}<25 \mathrm{G}<50 \mathrm{G}<$ $75 \mathrm{G}<100 \mathrm{G}$. Storage modulus of all hybrid composites is higher than unfilled neat epoxy. Neat epoxy comprises of only the matrix which gives material more flexibility resulting in a decreased stiffness which results in low storage modulus. When fibres are incorporated in the epoxy matrix, stiffness of the composites increases resulting in enhanced storage modulus. Also addition of fibres to matrix allows greater stress transfer at interface which consequently increases the storage modulus. Among the hybrid composites, composites with higher glass content have shown higher storage modulus due to hybrid effect caused by the presence of stiffer glass fibres. Roystonea regia fibres due to 
Table 3. Loss modulus and damping values of composites.

\begin{tabular}{lcccc}
\hline Sample & $\begin{array}{c}T_{\mathrm{g}}\left({ }^{\circ} \mathrm{C}\right) \text { obtained from } \\
\text { loss modulus curve }\end{array}$ & $\begin{array}{c}\text { Loss modulus }(\mathrm{MPa}) \\
\left(\tan \delta_{\max }\right)\end{array}$ & $\begin{array}{c}\text { Temperature }\left({ }^{\circ} \mathrm{C}\right) \text { at } \\
\tan \delta_{\max }\end{array}$ \\
\hline NE & 63.96 & 327.36 & 0.84 & 83.4 \\
OG & 67.72 & 341.56 & 0.79 & 87.2 \\
$25 \mathrm{G}$ & 71.46 & 412.52 & 0.75 & 83.1 \\
$50 \mathrm{G}$ & 75.21 & 425.64 & 0.73 & 84.2 \\
$75 \mathrm{G}$ & 79.22 & 453.92 & 0.64 & 87.2 \\
$100 \mathrm{G}$ & 83.40 & 470.23 & 0.57 & 83.6 \\
\hline
\end{tabular}

their higher hydrophilic nature possess lower stiffness when compared with glass fibre.

3.2b Loss modulus: Loss modulus for neat epoxy and different hybrid composites have been plotted as a function of temperature in figure 7 and table $3 . T_{\mathrm{g}}$ of all hybrid compo-

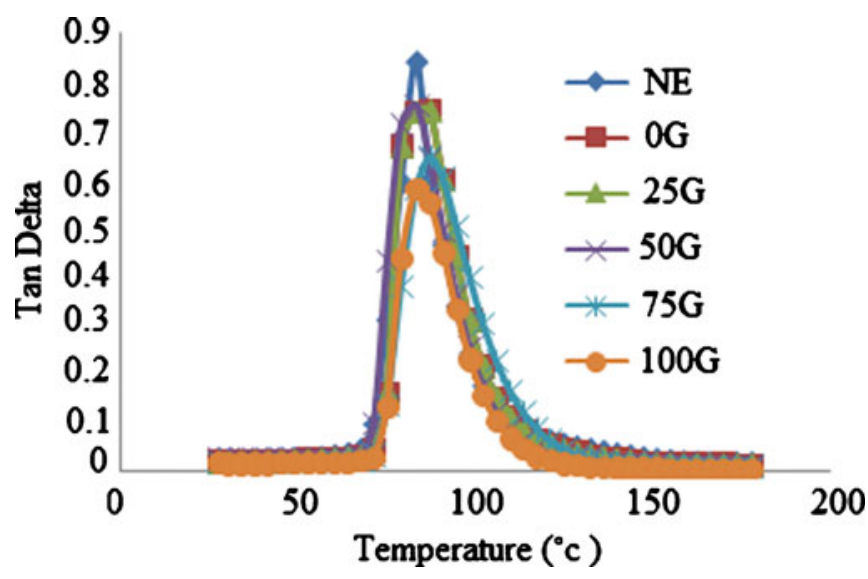

Figure 8. Effect of surface treatment on $\tan \delta$ at different temperatures. sites shifted to higher temperatures when compared with neat epoxy. It is due to the decreased mobility of matrix chains owing to the presence of the fibres. From the figure, loss modulus increased in the order $\mathrm{NE}<0 \mathrm{G}<25 \mathrm{G}<50 \mathrm{G}<$ $75 \mathrm{G}<100 \mathrm{G}$ indicating increase in loss modulus with the increase of glass content. This is due to the fact that in a hybrid composite, properties of the composite depend on the modulus and \% elongation at break of the individual reinforcing fibres. The strength and modulus of glass fibre is much higher than the strength and modulus of natural fibre. Hence hybrid composites with higher glass content would offer resistance against mobility of the matrix chains leading to higher loss modulus.

3.2c Tan $\delta$ : The variation of $\tan \delta$ of the neat epoxy and different hybrid composites as a function of temperature is shown in figure 8 . From figure 8 and table 3 it is evident that neat epoxy has the highest $\tan \delta$ peak value indicating higher degree of mobility and therefore, better damping properties. Roystonea regia hybrid composites have shown decreased $\tan \delta$ values due to the fact that incorporation of fibres would act as barriers and check mobility of the matrix chains. Reduction in matrix volume required for the dissipation of vibration energy after reinforcement is another reason. Also values indicate that hybrid composites with higher

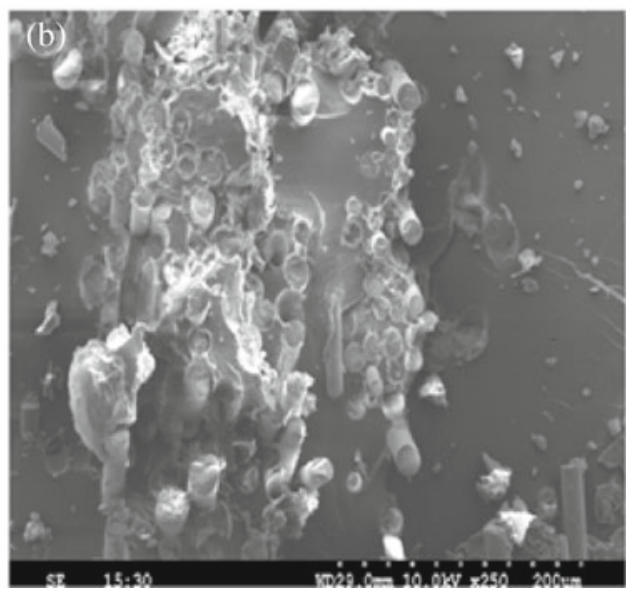

Figure 9. Scanning electron micrographs of tensile fractured surfaces of (a) 25G hybrid composites and (b) $75 \mathrm{G}$ hybrid composites. 
glass fibre content have shown decreased $\tan \delta$ values. As natural fibres possess less strength than glass fibres, strong and rigid interface develops between fibre and matrix in composites with higher glass content. This would reduce the molecular mobility in the interface region leading to lower damping values. The fracture morphology of tensile specimens was shown to compare the fibre/matrix interface of $25 \mathrm{G}$ and $75 \mathrm{G}$ composites in figures 9(a) and (b). From SEM, it is evident that $75 \mathrm{G}$ (figure 9(b)) has stronger interface as compared to $25 \mathrm{G}$ (figure 9 (a)).

\section{Conclusions}

An investigation into the effect of surface modification and hybridization on dynamic mechanical properties of Roystonea regia/glass-epoxy composites was presented. Storage modulus and loss modulus of reinforced composites were found to increase when compared with neat epoxy due to reinforcement effect of fibres leading to strong and stiff interface. But $\tan \delta$ of neat epoxy is higher than the reinforced composites indicating large degree of mobility in pure epoxy. Storage and loss modulus values increased after surface modification treatments with simultaneous decrease in $\tan \delta$ values. Alkali treatment proved to be more effective on dynamic mechanical properties as compared to silane treatment. In hybrid composites as glass fibre being stiffer than the Roystonea regia fibre, storage and loss modulus values increased with increase in glass fibre content while $\tan \delta$ values were found to decrease.

\section{References}

Bisanda E T N and Ansell M P 1991 Compos. Sci. Technol. 41 165

Bledzki A K and Gassan J 1999 Prog. Polym. Sci. 24221

Huda Masud S, Drzal Lawrence T, Mohanty Amar K and Misra Manjusri 2008 Compos. Sci. Technol. 68424

Jacob Maya, Francis Bejoy, Thomas Sabu and Varughese K T 2006 Polym. Compos. 27671

Kuzak S G and Shanmugam Arun 1999 J. Polym. Sci. 73649

Li Yan, Mai Yiu-Wing and Ye Lin 2000 Compos. Sci. Technol. 60 2037

Manikandan K C, Nair S M D and Thomas S 1996 J. Appl. Polym. Sci. 601483

Pickering K L, Beckermann G W, Alam S N and Foreman N J 2007 Compos. Part A $\mathbf{3 8} 461$

Pothan L A, Thomas S and Neelakantan N R 1997 J. Reinf. Plast. Compos. 16744

Raghavendra Rao H, Varada Rajulu A, Ramachandra Reddy and Hemachandra Reddy K 2010 J. Reinf. Plast. Compos. 291446

Rong Min Zhi, Zhang Ming Qiu, Liu Yuan, Yang Gui Cheng and Zeng Han Min 2001 Compos. Sci. Technol. 611437

Rout J, Misra M, Thripathy S S, Nayak S K and Mohanty A K 2001 Compos. Sci. Technol. 611303

Sinha E and Rout S K 2009 Bull. Mater. Sci. 3265

Srikala M S, George Jayamol, Kumaran M G and Thomas Sabu 2002 Compos. Sci. Technol. 62339 